\title{
3D Building Façade Reconstruction Using Handheld Laser Scanning Data
}

\author{
F. Sadeghi ${ }^{a^{*}}$, H. Arefia ${ }^{\mathrm{a}}$ A. Fallah ${ }^{\mathrm{a}}$ and M. Hahn ${ }^{\mathrm{b}}$ \\ a School of Surveying and Geospatial Engineering, University of Tehran, Tehran, Iran-fatemehsadeghi@ut.ac.ir
}

(alaleh.fallah, hosein.arefi)@gmail.com

b Stuttgart University of Applied Sciences, Stuttgart, Germany - Michael.hahn@hft-stuttgart.de

KEY WORDS: 3D Modelling, Grammar-based algorithm, Point cloud, Density histogram, RANSAC

\begin{abstract}
:
3D The three dimensional building modelling has been an interesting topic of research for decades and it seems that photogrammetry methods provide the only economic means to acquire truly 3D city data. According to the enormous developments of 3D building reconstruction with several applications such as navigation system, location based services and urban planning, the need to consider the semantic features (such as windows and doors) becomes more essential than ever, and therefore, a 3D model of buildings as block is not any more sufficient. To reconstruct the façade elements completely, we employed the high density point cloud data that obtained from the handheld laser scanner. The advantage of the handheld laser scanner with capability of direct acquisition of very dense 3D point clouds is that there is no need to derive three dimensional data from multi images using structure from motion techniques. This paper presents a grammar-based algorithm for façade reconstruction using handheld laser scanner data. The proposed method is a combination of bottom-up (data driven) and top-down (model driven) methods in which, at first the façade basic elements are extracted in a bottom-up way and then they are served as pre-knowledge for further processing to complete models especially in occluded and incomplete areas. The first step of data driven modelling is using the conditional RANSAC (RANdom SAmple Consensus) algorithm to detect façade plane in point cloud data and remove noisy objects like trees, pedestrians, traffic signs and poles. Then, the façade planes are divided into three depth layers to detect protrusion, indentation and wall points using density histogram. Due to an inappropriate reflection of laser beams from glasses, the windows appear like holes in point cloud data and therefore, can be distinguished and extracted easily from point cloud comparing to the other façade elements. Next step, is rasterizing the indentation layer that holds the windows and doors information. After rasterization process, the morphological operators are applied in order to remove small irrelevant objects. Next, the horizontal splitting lines are employed to determine floors and vertical splitting lines are employed to detect walls, windows, and doors. The windows, doors and walls elements which are named as terminals are clustered during classification process. Each terminal contains a special property as width. Among terminals, windows and doors are named the geometry tiles in definition of the vocabularies of grammar rules. Higher order structures that inferred by grouping the tiles resulted in the production rules. The rules with three dimensional modelled façade elements constitute formal grammar that is named façade grammar. This grammar holds all the information that is necessary to reconstruct façades in the style of the given building. Thus, it can be used to improve and complete façade reconstruction in areas with no or limited sensor data. Finally, a 3D reconstructed façade model is generated that the accuracy of its geometry size and geometry position depends on the density of the raw point cloud.
\end{abstract}

\section{Introduction}

The three dimensional building modelling has been an interesting topic of research for decades and it seems that photogrammetry provides the only economic means to acquire truly 3D city data (Förstner, 1999). According to the enormous developments of 3D building reconstruction with several applications such as navigation system, location based service, and urban planning, the need to consider the semantic features (such as windows and doors) becomes more essential than ever, and therefore, a 3D model of building as block is not any more sufficient and the generation of the three dimensional detailed models is still an open question that needs research yet.

\subsection{Related works}

We can classify the modelling methods according to type of input data. The first class for 3D façade modelling is the image based modelling. It is a classic topic research and many academicians tender several methods. Ripperda used single image to detect façade elements and named terminals that used for façade grammar. Facade grammar describes the façade style. Reconstruction of façade is a stochastic process of interpreting a sensor data with the grammar (Ripperda, 2006). Cornelis used essential video frames, then dense matching between stereo frames had done, but in this method building boundary were not be determined and this method just focused on the visualization (Cornelis, 2008). Frueh used depth image that it was obtained from point cloud, then the histograms were plotted in order to distinguish façade points from irrelevant objects, then the façade points were used to generate the TIN mesh models and the texture of photos mapped to models (Frueh, 2005). Pu segmented the building façade points and classified the data to several clusters. This is done by planner surface growing algorithm, and all extracted features will be recognized by their property such as, height, position, topology and etc. These properties are inferred from human knowledge (Pu, 2009). Yousefzadeh classified the façade point cloud data into three depth layers. The internal layer that contained all the information about façade elements was stored for next processes, then the mentioned layer was rasterized in order to splitting and extracting façade features (Yousefzadeh, 2014). Becker represented a fully automatic façade reconstruction that includes both bottom-up and topdown modelling. The proposed method starts with searching for holes to determine the windows and other components, then the output model was used for inferring the façade grammar. Doors and windows constitute the vocabularies of the grammar and rules will be inferenced by detecting the relation between these 
basic elements, then the façade grammar will be used to reconstruct vacant facades (Becker, 2009).

\subsection{Overview of algorithm}

At first, the façade plane is detected and then it is decomposed to find the internal plane which contains façade elements using depth histogram. Then the rasterized inner plane is partitioned to extract structures, and the depth values are added to features using histogram. After bottom up modelling, the repeated components are detected and then clustered to find hierarchical interrelationship between them in order to infer the rules. Finally, the façade grammar that contains 3D basic elements and rules are used to complete the rest of façade. Figure 1 shows a flowchart of the grammar-based façade reconstruction with two bottom-up and top-down modelling steps. The following subsections are discussed each step of flowchart in details.

\subsection{Dataset}

The point cloud data that is used acquired by handheld laser scanner Zebedee, it is consist of $2 \mathrm{~d}$ laser scanner and an inertial measurement unit mounted on one or two springs, it has 270 degrees field of view. Its mass is $210 \mathrm{~g}$ which makes it ideal for low measurement and it is maximum range is $30 \mathrm{~m}$.

\section{Plane Detection}

The captured data by handheld laser scanner not only have information about buildings and façade planes but also irrelevant objects such as tree, sign traffic, pedestrians and etc. are distinctive. So the façade plane must be separated from other objects. For this, the conditional RanSAC is used to detect walls in a way of keeping the windowsill and niches information, detect the major planes and increase the robustness of method. Figure 2 shows depth histogram that the highest peak is relative to main wall points, and the left peak shows the inner plane with façade elements points.

\section{Cutting Out Non-Quality Data}

Because the low range of Zebedee, upper parts of façade were not scanned and also obstacles cause occluded area, the point cloud density is not homogenous and causes to bad influence on output model. Due to uncertainty and lack of details at top of façade plane, the properties and characteristics of basic elements cannot be inferred, so the low density parts are eliminated and the lower parts of façade plane with an acceptable density is just used. At first, the density histogram is plotted in both $\mathrm{x}$ and $\mathrm{y}$ direction to find the range which the number of points will decrease abruptly. After that, the data are clipped based on this range and used for the further process. Figure 3 shows the depth image that was used for clipping the dense data.

\section{Decomposing Façade Plane}

In this step, a histogram is plotted to find out the distribution of point cloud perpendicular to façade plane. This histogram have normally three picks that they indicate the protrusion, wall and indentation ranges. The inner points indicate the information about windows and doors (façade elements). A plane is fitted on points that situate in internal range and the next processes will be done on this plane.

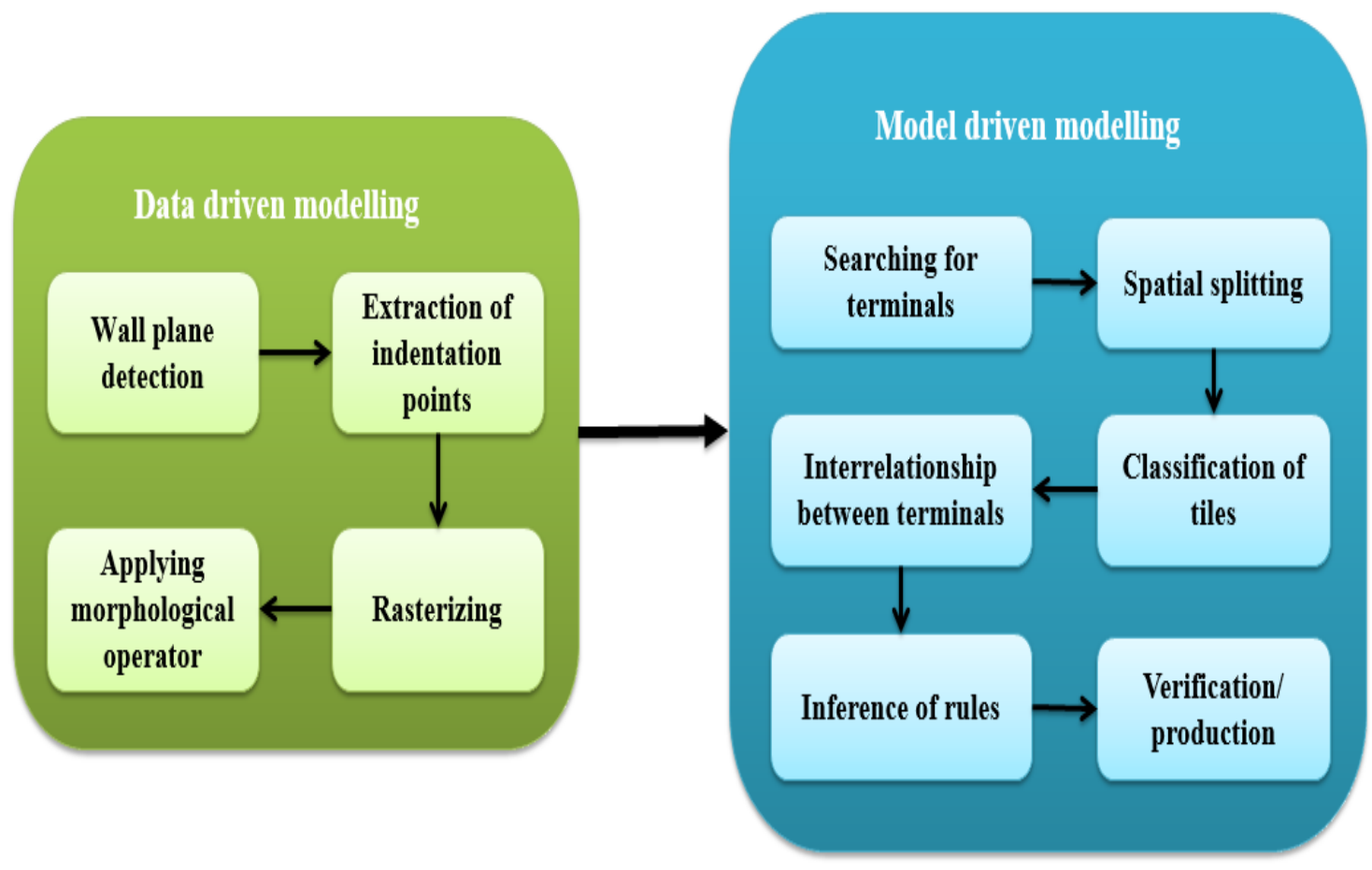

Figure 1. Processing flowchart of the grammar based façade reconstruction 


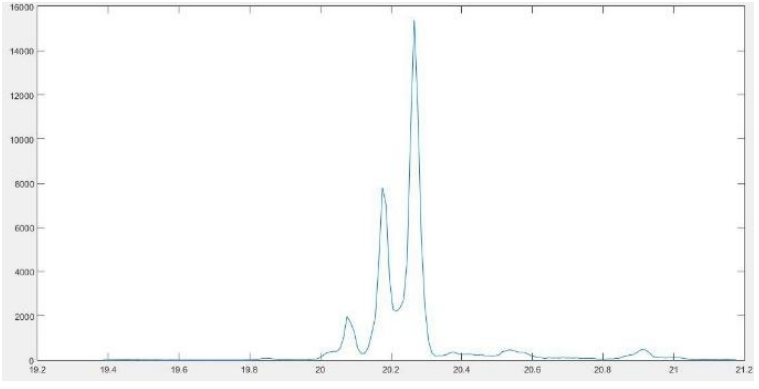

Figure 2. The depth histogram

\section{Rasterizing}

In this step, the point cloud data is converted to low resolution binary image. For this, a two dimensional grid is created on façade points. The size of grid element is arbitrary and should be chosen by user. Each cell may contain point or not, that results in one and zero values, respectively. Binary image makes it possible to remove noises by image processing's tools. Figure 4 shows the rasterized inner plane.

\section{Feature Detection}

For feature detection, the sum of values of each row and column are calculated separately. Based on the obtained values, the vertical and horizontal histograms are plotted to determine the floors and basic elements of façade, respectively. It means that rows with minimum values in vertical histogram are probably the best candidate that floor separator lines pass through them must pass. Figure 5.a shows the horizontal histogram and Figure 5.b shows the smoothed one that it is easier to find the minimums. So each floor has the particular horizontal histogram. The columns with zero values of this histogram are selected and then a line passes through the middle of these adjacent columns. Figure 6.a and figure 6.b shows the vertical histogram and smoothed histogram for each floor, respectively. The advantage of our method related to previous researches is that there is no need to move the lines and change their positions. Figure 7 shows the partitioned façade without noise and extracted features.

After reticulation, each cell has a feature that the noise and isolated pixels are removed and the openings borders are refined by morphological operators. Then the searching process is started to find the pixels with one value. The pixels with the minimum and maximum row and column is used to discover the range of features. Then a rectangle is fitted to them. In proposed method, we can compute the planner coordinates of features and for determining depth we use the histogram that generated in section 4 .

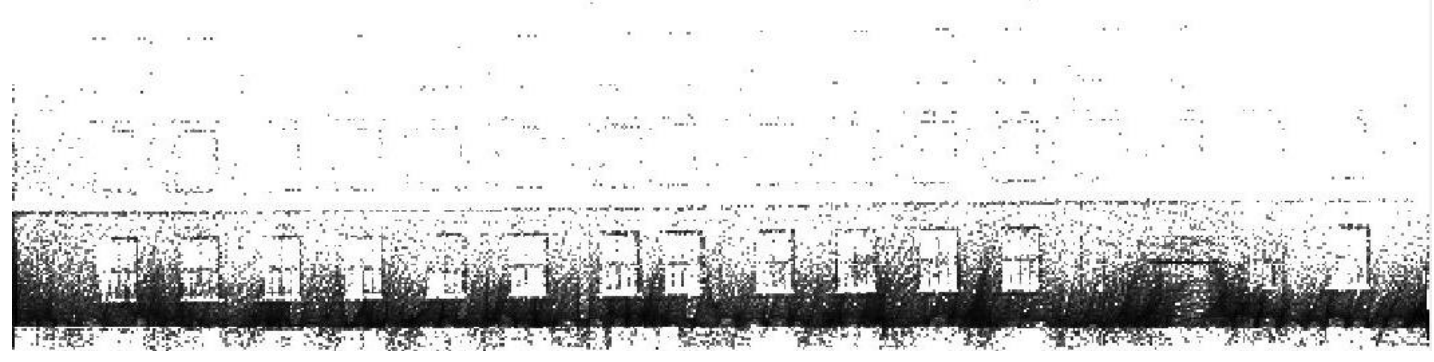

Figure 3. The depth image

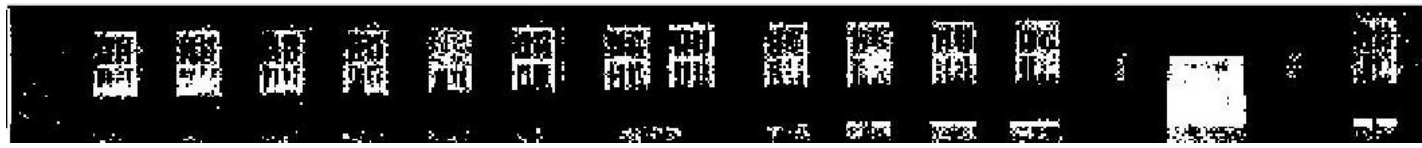

Figure 4. The rasterized point cloud

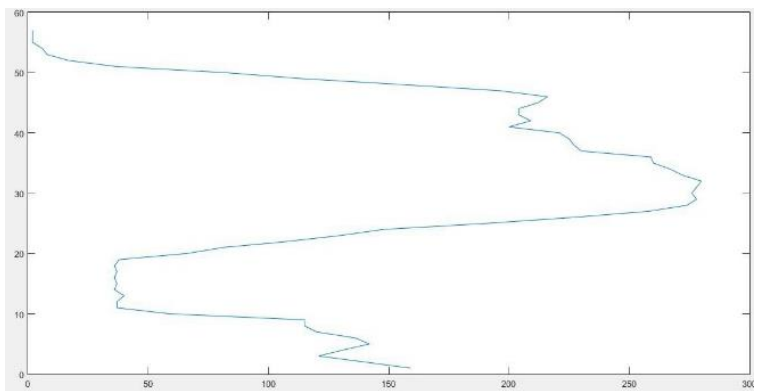

Figure 5.a. The horizontal histogram

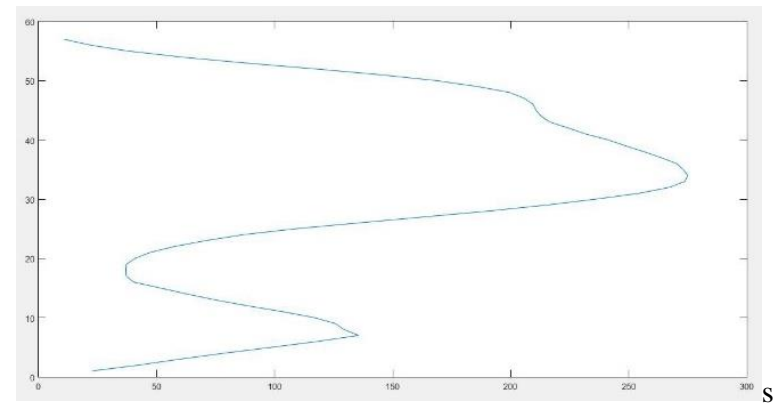

Figure 5.b. The smoothed horizontal histogram 


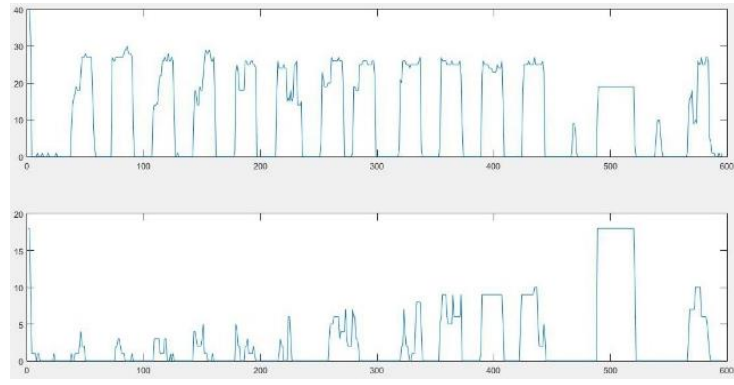

Figure 6.a. The vertical histogram for each floor

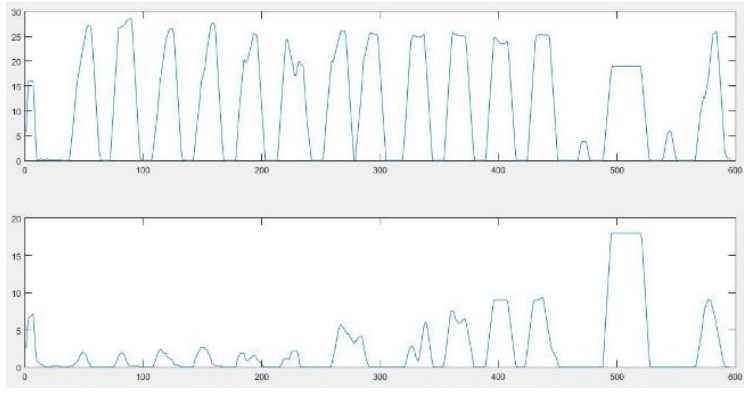

Figure 6.b. The smoothed vertical histogram for each floor

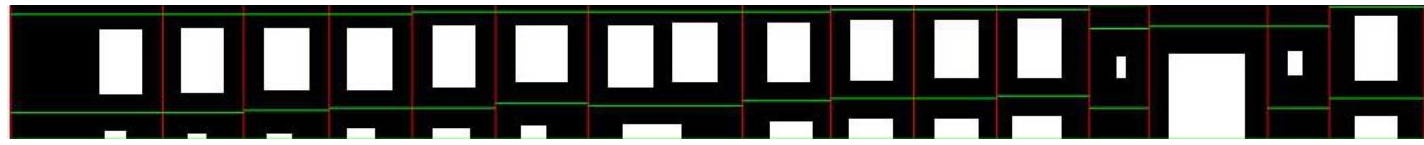

Figure 7. Partitioned façade without noise and extracted features

\section{Façade Grammar}

The output model of data-driven reconstruction has all the information and is the base for further process in top down modelling. The façade grammar describes the architectural appearance of façade and can add details to areas with no sensor data. It can also modify façade in areas with non-accurate data. Indeed façade grammar is composed of vocabularies or terminals $(\mathrm{T})$, production or replacement rules $(\mathrm{R})$, non-terminals that can be replaced by terminals $(\mathrm{N})$ and vacant façade that is a starting point for substitution that named $\mathrm{V}$, and the façade elements constitute the vocabulary of the grammar and the interrelationship between the vocabs form the rules.

The rest of the process is divided to three steps; at first step, the basic component of façade that are known as geometry tiles must be identified and their properties such as width and height are stored, blank space between geometries are considered as wall tiles and just the width property will be saved for this kind of elements. The second step is formation of higher order structure from terminals and inferring the rules and the last step is about completion of façade and adding façade elements to areas with no data.

\subsection{Floor determination}

The binary image that was created in section 5, is used to diagnose the floors. The arrangement of geometries effects on determination of floors, i.e. if upper side of geometries are approximately in a line, the rows with zero values are chosen, then a line is passed through the middle of these adjacent rows. For the case one of geometries is shifted vertically and top side of geometries aren't in a line, the same work is done for columns, then the area between two vertical delimiter is just considered. Floor lines are specified then those situated near each other are merged. Figure 8 shows façade and separator horizontal lines.

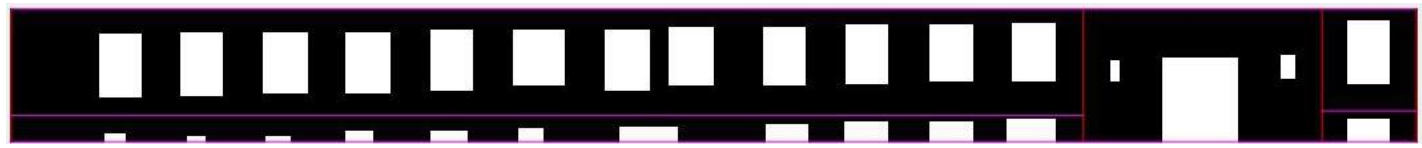

Figure 8. Detected floors using vertical and horizontal lines

\subsection{Detection of terminals}

As mentioned before, the basic irrefrangible elements of façade form the vocabularies of the grammar. Detection of geometries is based on shifting vertical line for each floor to find changes in binary image and then geometries, walls and their width will be stored for each floor. Height is an additional property which is just stored for the geometries.

\subsection{Clustering the tiles}

Due to the large volume that was occupied by information, the information should be summarized by clustering the tiles and grouping them. Classification of wall tiles is based on width property and walls with same width will be situated in a group, while for clustering the geometry tiles, depth image is used. For this aim, we lay a two dimensional grid over the façade points. The grid elements size is a bit larger than point sampling size. For cells with more than one point, the nearest point to the façade plane and its perpendicular distance as its pixel value are considered. For the cell with no point, the pixel value is set zero, and then the pixel values are normalized into the range $[0,1]$. For classification, the correlation values of depth images are evaluated, and a threshold is determined, so if correlation value is higher than the threshold, two geometry are in a same cluster.

\subsection{Interrelationship between terminals}

As a result of previous steps, the higher order structures from detected tiles are obtained, so the SEQUITUR (Nevill-Manning. 1997) is used to detect the relationship between components and discrete symbols. It can find the pattern and regularities without referencing to the meaning of individual symbol and finally compress the sequence. It can find the hierarchical interrelationship between terminals in terms of rewrite rules. However, our algorithm is different in three concepts contrast to SEQUITUR to remove repeated symbols. 
1. The proposed method is able to remove three adjacent symbols in the form of gwg ( $\mathrm{g}$, w is the symbol of geometry terminal and wall terminal respectively) contrast to SEQUITUR that it just considers two adjacent symbols.

2. No triples occur more than once while the SEQUITUR removes the replaced structure that appears once.

3. The proposed method first prioritize triplets with small wall terminal but in SEQUITUR there is no priority.

So, the algorithm is applied in order to compress the tile sequences, this is done fully automatically until there is no repeated triple ( $\mathrm{gwg})$.

The hierarchical interrelationship between terminals can be stored, and each terminal and structure can have a hierarchical value. Terminals and structures in compressed string will have the highest value and lower order structures and terminal will get lower hierarchical value gradually.

\subsection{Rule inference}

Based on sets of terminals and non-terminals, rules which are the most important parts of façade grammar can be inferred. In rest of subsection the rules are categorized and introduced in details.

The rules $\mathrm{p}_{1}$ and $\mathrm{p}_{2}$ corresponds to horizontal and vertical partitioning of façade, rule $\mathrm{p}_{1}$ divides façade into floors, so the horizontally partitioned façade is indicated by Ws. Floor is split into tiles by rule $\mathrm{p}_{2}$, and it substitutes the floor to the sequence of tiles in the form of wall tile, geometry tile and wall tile. Rule $\mathrm{p}_{3}$ substitute geometry non-terminal to structures that explained section 7.4. Geometries and wall terminals produce rules $\mathrm{p}_{4}$ and p5, respectively. The first condition is to ensure that there is enough space in façade, and second condition in rule $\mathrm{p} 5$ is for preventing generate structures, for example the wall tile $\mathrm{w}_{\mathrm{i}}$ which is part of structure $S_{k}$ cannot be nearby $S_{k}$.

\section{$P_{1}: V \rightarrow W s$}

\section{$\mathrm{P}_{2}: \mathrm{W} \rightarrow \mathrm{WGW}$}

If width $(\mathrm{W}) \geq$ width $(\mathrm{WGW})$

$\mathrm{P}_{3}: \mathrm{G} \rightarrow \mathrm{Si}: \mathrm{P}\left(x \mid \mathrm{p}_{3}\right)$

If width $(\mathrm{G}) \geq$ width $\left(\mathrm{S}_{\mathrm{i}}\right)$

$\mathrm{P}_{4}: \mathrm{G} \rightarrow \mathrm{g}_{\mathrm{i}}: \mathrm{P}\left(x \mid \mathrm{p}_{4}\right)$

If width $(\mathrm{G}) \geq$ width (gi)

$\mathrm{P}_{5}: \mathrm{W} \rightarrow \mathrm{w}_{\mathrm{i}}: \mathrm{P}\left(x \mid \mathrm{p}_{5}\right)$

width $(\mathrm{W}) \geq$ width $\left(\mathrm{w}_{\mathrm{i}}\right) \& \&$

hierarchy left-context $\leq$ left_hierarchy $w_{i} \& \&$

hierarchy right-context $\leq$ right_hierarchy $\mathrm{w}_{\mathrm{i}}$

\section{Production}

As mentioned before, we use just dense areas to extract features and infer the grammar, then remaining parts of façade should be enriched during production process.
At first, wi terminals at façade borders are replaced by nonterminals, then the geometry non terminal is selected in the string and a rule with this non terminal is chose. After that, the terminals are substituted by non-terminals and it is repeated until all terminals are situated, otherwise the process will be continued by another non-terminal. Sometimes, there is more than one rule to replace nonterminal, so it needs to determine the probability of occurrence of each rule at any position, and select the rule with highest probability. The $\left(\boldsymbol{p}_{i} \mid \boldsymbol{x}\right)$ is calculated as follows:

$$
\mathrm{P}\left(p_{i} \mid x\right)=\frac{\mathrm{P}\left(x \mid \mathrm{p}_{i}\right) \mathrm{P}\left(p_{i}\right)}{\mathrm{P}(x)}
$$

Where $\boldsymbol{P}\left(\boldsymbol{p}_{i} \mid \boldsymbol{x}\right)$ denotes the required posterior probability of the rule pi for a given position $\mathrm{x}$ on the façade. $\boldsymbol{P}\left(\boldsymbol{p}_{\boldsymbol{i}}\right)$ is the probability for the occurrence of $\boldsymbol{p}_{\boldsymbol{i}}$ that it can approximated by hierarchical of rules.

The $\boldsymbol{P}(\boldsymbol{x})$ is marginal probability of $\boldsymbol{x}$

Figure 9 shows the data-driven output model that is restricted to dense data, figure 10 shows the grammar based model that has predicted features in no data area. It is obvious non reconstructed features during data-driven modelling are not predicted in grammar based façade reconstruction.

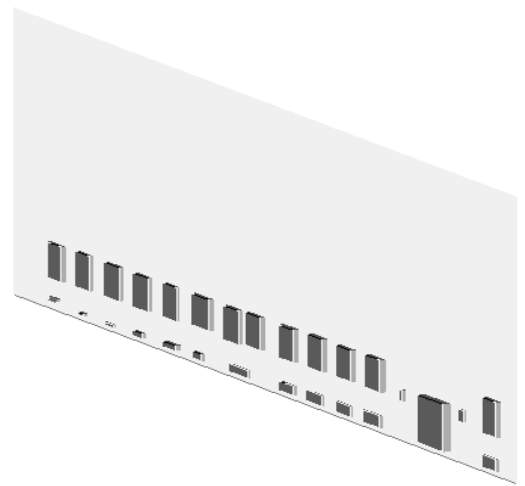

Figure 9. The data driven output model

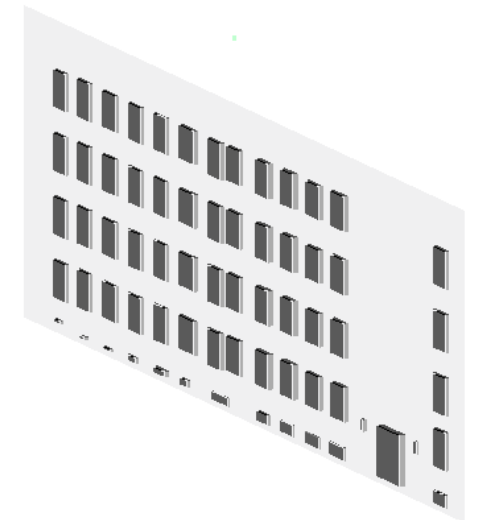

Figure 10. The grammar-based output model

\section{Conclusion}

Because of some data acquisition conditions, such as occluded area and non-complete data of the upper floors, having a 
homogeneous dense data for façade reconstruction is not possible. In this paper, a grammar based façade reconstruction was implemented. We restricted data-driven modelling to areas with dense data and we just used the dense data related to lower floors, using dense parts of data caused to derivate vivid elements, and removing noise aims to preventing generate unrealistic features also using vertical histogram for each floor increase the automation level of data driven process, we extracted three dimensional structures from data driven output model, then the remaining part of façade was completed via inferenced grammar.

\section{References}

Becker, S., Haala, N., Fritsch, D., 2008. Combined Knowledge Propagation for Facade Reconstruction. IAPRS \& SIS Vol. 37 (B5), pp. 1682-1750

Becker, S. and Haala, N. 2009, Grammar Supported Facade Reconstruction from Mobile LIDAR Mapping. In Proc. CMRT (XXXVIII, Part 3/W4; Paris, France: Int. Arch. Photogramm., Remote Sens. Spatial Inf. Sci), 229-234.Becker, S., 2009. Generation and application of rules for quality dependent facade reconstruction. ISPRS Journal of Photogrammetry and Remote Sensing, 64(6), pp. 640-653.

Bosse, M., Zlot, R. and P. Flick. Zebedee: Design of a spring mounted 3D range sensor with application to mobile mapping. IEEE Transactions on Robotics, 28(5), pp. 1104-1119

Cornelis, N., Leibe, B., Cornelis, K., Gool, L., 2008. 3d urban scene modeling integrating recognition and reconstruction. International Journal of Computer Vision Vol. 78, No. 2-3, pp. 121-141

Forstner, W., 1999. 3D-city models: Automatic and semiautomatic acquisition methods. In: D. Fritsch and R. Spiller (eds), Photogrammetric Week 99, Wichmann Verlag, pp. 291303

Frueh, C., Jain, S. and Zakhor, A. 2005. Data processing algorithms for generating textured $3 \mathrm{~d}$ building facade meshes from laser scans and camera images. Int. J. Comput. Vision, 61(2), pp. 159-184.

Haala, N., Becker, S., Kada, M., 2006. Cell Decomposition for the Generation of Building Models at Multiple Scales. IAPRS Vol. 36 (3), pp. 19-24

Schnabel, R., Wahl, R., and Klein, R. 2007, Efficient RANSAC for Point-Cloud Shape Detection, Computer Graphics Forum, 26 (2), pp. 214-26.

Ripperda, N. and Brenner, C. 2009, Application of a Formal Grammar to Facade Reconstruction in Semiautomatic and Automatic Environments, AGILE International Conference on Geographic Information Science

$\mathrm{Pu}$, S. and Vosselman, G. 2009, Knowledge based reconstruction of building models from terrestrial laser scanning data, ISPRS Journal of Photogrammetry and Remote Sensing, 64 (6), pp. $575-84$.
Nevill-Manning. 1997. Compression and explanation using hierarchical grammars. The Computer Journal 40 (2-3), pp. 103116.

Ripperda, N., Brenner, C., 2006. Reconstruction of façade structures using a formal grammar and rjMCMC. In: Proceedings of the 28th Annual Symposium of the German Association for Pattern Recognition DAGM 2006. pp. 750-759.

Yousefzadeh, M., Leurink, F., Beheshti jou, M., 2014. A general data driven algorithm for façade structure modelling using ground based laser data. The International Archives of the Photogrammetry, Remote Sensing and Spatial Information Sciences, Volume XL-3, ISPRS Technical Commission III Symposium, pp. 381-386. 